average $(\mathrm{H}=637.1 ; \mathrm{p}<0.001)$. When considering an 'ideal' setting, clinicians in South America $18 \%$ and South Asia $19.5 \%$ were most likely to add EVT versus the $6.9 \%$ global average.

Conclusion In the selection of patients to undergo endovascular treatment, there exists a significant difference in rate of EVT procedures performed across countries and regions in the world reflecting local resources and practice settings. The decision to proceed with EVT reflects factors such as center volumes and clinician age $\&$ experience as contributing factors with younger clinicians in centers with lower volumes being more likely to consider EVT in an 'ideal' setting.

Disclosures N. Kashani: None. J. Ospel: None. M. Almekhlafi: None. A. Wilson: None. A. Rabinstein: None. W. Kunz: None. B. Campbell: None. M. Foss: None. F. Turjman: None. U. Fischer: None. B. Baxter: None. P. Sylaia: None. S. Yoshimura: None. J. Heo: None. B. Kim: None. M. Hill: None. G. Saposnik: None. B. Menon: None. M. Goyal: None.

\section{E-094 EFFECT OF DEFINITION AND METHODS ON ESTIMATES OF PREVALENCE OF LARGE VESSEL OCCLUSION IN ACUTE ISCHEMIC STROKE: SYSTEMATIC REVIEW}

${ }^{1} \mathrm{M}$ Waqas*, ${ }^{2} \mathrm{~A}$ Rai, ${ }^{1} \mathrm{~K}$ Vakharia, 'A Siddiqui. ${ }^{1}$ UB Neurosurgery, Buffalo, NY; ${ }^{2}$ Radiology, West Virginia University Hospital, Morgantown, WV

\subsection{6/neurintsurg-2019-SNIS.169}

Background An accurate estimate of large vessel occlusion (LVO) incidence is critical for planning stroke systems of care and approximating workforce requirements. The purpose of this systematic review was to estimate LVO prevalence among acute ischemic stroke (AIS) patients with emphasis on definitions and methods used by different studies.

Methods Using Medical Subject Headings terms, EMBASE, PubMed, Web of Science, Google Scholar, and the Cochrane Library were searched for prevalence of LVO and AIS from 2000-2018. All articles in the English literature describing the frequency of LVO among AIS patients were included in this review. Studies without consecutive recruitment or without confirmation of LVO with computed tomography angiography or magnetic resonance angiography were excluded. Studies were grouped based on similarities in LVO definition and methods.

Results Eighteen records met the eligibility criteria. Five studies presented population-based estimates and 13 provided single-hospital experiences (5 prospective, 8 retrospective). The AIS denominator (number of all AIS) from which LVO rates were generated was variable. Nine different definitions were used, based on occlusion site. The prevalence of LVO among suspected AIS patients ranged from 13-46\%. The overall prevalence was 29.1\% (95\% CI, 21.5-36.77). Pooled prevalence of LVO among suspected AIS patients in population-based studies was $27.10 \%$ (95\% CI 19.46-34.74); for studies defining AIS based on magnetic resonance imaging, it was $21.49 \%$ (95\% CI 13.8-29.1).

Conclusion LVO definition and AIS denominator greatly influence estimates of LVO prevalence among AIS patients. Population-based studies with standardized methods must be conducted to provide data for planning and developing stroke systems of care.
Disclosures M. Waqas: None. A. Rai: 2; C; Medtronic, Cerenovus. K. Vakharia: None. A. Siddiqui: 1; C; Co-investigator NIH/NINDS 1R01NS091075. 2; C; Amnis Therapeutics, Boston Scientific, Canon Medical Systems USA Inc, Cerebrotech Medical Systems, Cerenovus, Corindus Inc., Endostream Medical Ltd, Guidepoint Global Consulting, Imperative Care, Integra LifeSciences Corp, Medtronic, MicroVention, Q’Apel Medical Inc, Rapid Medical, Rebound Therapeutics Corp., Serenity Medical Inc, Silk Road Medical, StimMed, Stryker, Three Rivers Medical, VasSol, W.L. Gore \& Associates. 4; C; Amnis Therapeutics, Apama Medical, Blink TBI Inc, Buffalo Technology Partners Inc, Cardinal Consultants, Cerebrotech Medical Systems, Cognition Medical, Endostream Medical Ltd, Imperative Care, International Medical Distribution Partners, Neurovascular Diagnostics Inc, Q'Apel Medical Inc, Rebound Therapeutics Corp, Rist Neurovascular In, Serenity Medical, Silk Road Medical, StimMed, Synchron, Three Rivers Medical Inc, Viseon Spine.

\section{E-095 BGC PROTECTED ADAPT REDUCED RISK OF DISTAL EMBOLIZATION: AN IN VITRO STUDY}

R Arslanian*, M Marosfoi, C Raskett, MJ Gounis, AS Puri, J Chueh. New England Center for Stroke Research, UMass Medical School, Worcester, MA

\subsection{6/neurintsurg-2019-SNIS.170}

Introduction Recent studies have demonstrated that a direct aspiration first pass technique (ADAPT) for the treatment of acute ischemic stroke may be effective and safe. In this study, we sought to optimize the ADAPT technique by incorporating an adjunctive device such as a balloon guide catheter (BGC). The impact of diameter of the aspiration catheter as well as use of BGC on risk of distal embolization and rate of first pass success was evaluated.

Methods Mechanical thrombectomy was simulated in an invitro Circle of Willis model. To create a challenging occlusive burden in the MCA, a $20 \mathrm{~mm}$ long clot analog was prepared. Direct aspiration was applied via an 0.068 distal access catheter (DAC, Cat 7, Stryker Neurovascular, group: ADAPT068), a large 0.074' DAC (AXS Vecta 074, Stryker Neurovascular, group: ADAPT074) or 0.068 ' DAC with a BGC (FlowGate 2 Balloon Guide Catheter) placed at the cervical ICA (group: ADAPT068-BGC). In both the ADAPT068 and 074 groups, a long 0.091' sheath was used in conjunction with the DAC. The pump was turned on before the aspiration catheter was advanced proximal to the clot. Each run was assigned a TICI score to assess degree of recanalization. Ten replicates were performed per group, and the maximum thrombectomy attempt was limited to three per run. Distal emboli greater than $200 \mu \mathrm{m}$ were collected and measured using the Coulter Principle.

Results The lowest number of distal emboli was observed when BGC was used in the ADAPT068 group. Collection of visible emboli greater than $1000 \mu \mathrm{m}$ yielded one particle from both the ADAPT068 and ADAPT068-BGC treatment groups, while ADAPT074 followed with four particles (average size $2.25 \mathrm{~mm}$ ). A total of 34 emboli sized between $200-1000 \mu \mathrm{m}$ were collected from the ADAPT068 group, and the number of emboli was reduced by more than $50 \%$ when a BGC was employed in the ADAPT068-BGC group $(\mathrm{P}>0.05)$. The highest rate of first pass recanalization was observed in the 


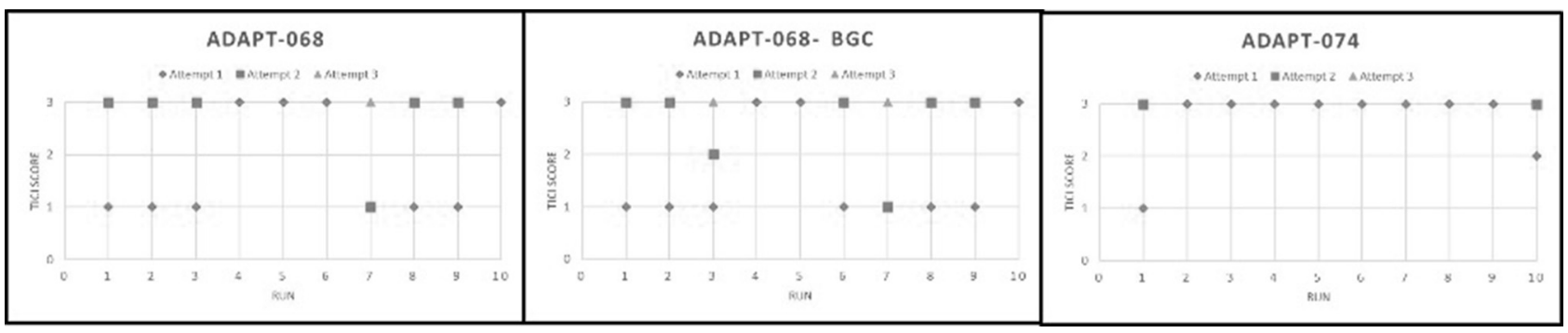

\section{Abstract E-095 Figure 1}

ADAPT074 treatment group with the largest bore catheter; where $80 \%$ of runs scored a TICI 3 after one attempt (see figure 1). Complete ingestion was observed in $90 \%$ of cases with the ADAPT074 technique, while the ADAPT068-BGC and ADAPT068 groups achieved rates of $70 \%$ and $60 \%$ respectively.

Conclusion BGC protected thrombectomy has been shown to reduce distal emboli for stent-retrievers previously. These preliminary data show that BGC protected thrombectomy also imparts benefit for ADAPT. The larger bore aspiration (ADAPT074) achieved the highest rate of first pass recanalization and complete clot ingestion.

Disclosures R. Arslanian: None. M. Marosfoi: 2; C; Stryker Neurovascular, InNeuroCo. Inc., ThrombX. C. Raskett: None. M. J. Gounis: 1; C; Cerenovus, Imperative Care, MIVI Neurosciences, Stryker Neurovascular, National Institute of Health (NIH), US-Israel Binational Science Foundation, Anaconda, Cook Medical, Gentuity, InNeuroCo, Magneto, Microvention, Medtronic Neurovascular, Neuravi, Neurogami, Philips Healthcare, Rapid Medical, Route 92 Medical, Syntheon, Wyss Institute. 2; C; Cerenovus, Imperative Care, MIVI, Phenox, Route 92 Medical, Stryker Neurovascular. 4; C; Imperative Care, Neurogami. A. S. Puri: 1; C; Medtronic Neurovascular, Stryker Neurovascular. 2; C; Medtronic Neurovascular, Stryker Neurovascular. J. Chueh: 2; C; Stryker Neurovascular, InNeuroCo. Inc.

\section{E-096 WHICH PATIENTS WITH PROXIMAL OCCLUSION STROKES MAY NOT GET REFERRED FOR ENDOVASCULAR THROMBECTOMY? INSIGHTS FROM AN INTERNATIONAL MULTIDISCIPLINARY SURVEY}

${ }^{1} \mathrm{~N}$ Kashani ${ }^{*},{ }^{2} \mathrm{~J}$ Ospel, ${ }^{1} \mathrm{~A}$ Wilson, ${ }^{1} \mathrm{M}$ Hill, ${ }^{1} \mathrm{~B}$ Menon, ${ }^{3} \mathrm{G}$ Saposnik, ${ }^{1} \mathrm{M}$ Goyal, ${ }^{4} \mathrm{M}$ Almekhlafi. 'University of Calgary, calgary, AB, Canada; ${ }^{2}$ University Hospital Basel, Basel, Switzerland; ${ }^{3}$ University of Calgary, Toronto, ON, Canada; ${ }^{4}$ University of Calgary, Calgary, $A B$, Canada

\subsection{6/neurintsurg-2019-SNIS.171}

Background The use of endovascular thrombectomy (EVT) is increasing in patients with large vessel occlusion (LVO) strokes. A few areas of uncertainty remain, and multiple trials are under way to address some of these questions. To guide future trials and to address practice gaps, knowledge of the cases where physicians are hesitant to offer EVT will be helpful.

Methods An international web-based survey was sent to practitioners in 38 countries to assess their decision-making toward selecting the management approach for LVO strokes in realworld. Ten case scenarios were randomly presented to each respondent from a pool of 22 cases. Participants were asked to choose whether they would offer EVT in that scenario in their current practice settings. We identified and summarized the cases in which the decision to perform EVT was significantly lower than the overall median for EVT use. We investigated the factors that may have influenced the decision to withhold EVT and the level of evidence for EVT in these cases according to the American Stroke Association guidelines. Results 607 physicians (mean age of 44 (SD 8.5) years, 83.5\% men, $53.6 \%$ neurologists, $28.7 \%$ neuro-interventionists, $13.3 \%$ neurosurgeons, $4.7 \%$ other) participated. The median number of participants who scored each scenario was 276. In the overall cohort, the median responses in favour of EVT in the participants' current practice setting were 78.7\%.

Ten scenarios received a significantly lower response in favour of EVT than the cohort median. These cases had a median EVT use of $60.3 \%$. Nine scenarios describe cases in which level 2B guideline recommendation for EVT exist while one scenario had a $1 \mathrm{~A}$ level of evidence.

The scenarios with low EVT use describe patients with poor baseline functional status (EVT use 66.4\%), patients with M2-occlusion (EVT use 61.3\%), mild clinical deficits (EVT use $60.4 \%$ ), baseline ASPECTS $\leq 4$ (EVT use 57\%), or with isolated intracranial carotid occlusion but patent MCA (EVT use $55.6 \%)$.

Conclusion Occlusion site, clinical symptoms severity, and low ASPECTS scores were determinant factors in the low responses in favour of EVT in our survey.

Disclosures N. Kashani: None. J. Ospel: None. A. Wilson: None. M. Hill: None. B. Menon: None. G. Saposnik: None. M. Goyal: None. M. Almekhlafi: None.

\section{E-097 EMERGENT CAROTID ARTERY STENTING IMPROVES NEUROLOGICAL OUTCOME IN PATIENTS WITH ACUTE ISCHEMIC STROKE}

K Kim*, K Jang. Neurosurgery, Incheon St. Mary's Hospital, The Catholic University of Korea, Incheon, Korea, republic of

10.1136/neurintsurg-2019-SNIS.172

Background and Purpose Emergent carotid artery stenting (CAS) is technically challenging and has concerns for clinical outcome and complications. Moreover, there is no definite guidelines for endovascular treatment of tandem lesion in acute ischemic stroke.

Materials and methods Multicenter retrospective study including three hospitals was performed. Forty-five patients with acute ischemic stroke by atherosclerosis of the extracranial carotid artery were enrolled. Mean age was 73.8 (59-98) and male to female ratio was 38:7. Mean initial NIHSS was 12.8 (4-28). Intravenous t-PA was used in 20 (44.4\%) patients. Mean initial stenosis of the ICA was $99.3 \%$. Favorable 